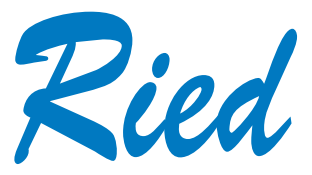

REVISTA IBEROAMERICANA DE EDUCACIÓN A DISTANCIA

Vol. 1 - № 2

Diciembre, 1998

\title{
Calidad de los Tutores en las Escuelas Residenciales: un Análisis Estadístico
}

\author{
(Tutor Quality at Residential Schools: A Statistical Analysis)
}

\author{
GORDON BURT \\ Open University \\ (Reino Unido)
}

\begin{abstract}
RESUMEN: La investigación sobre escuelas residenciales ha planteado la pregunta sumativa de si las escuelas son rentables y también la pregunta formativa de cómo pueden mejorarse. Es preciso utilizar una mayor sofisticación estadística en la investigación de la enseñanza a distancia. Se elaboran y prueban modelos estadísticos de los diversos aspectos de las escuelas residenciales. La principal diferencia entre los grupos de alumnos radica en las calificaciones que dichos alumnos conceden a la capacidad del tutor de su grupo. La actuación de un tutor a lo largo de los años se mantiene aproximadamente al mismo nivel. Un punto de diferencia en la escala en la calificación de la capacidad de un tutor está relacionado con una diferencia de medio punto en la calificación del valor global de la escuela. Se señala la repercusión organizativa de este trabajo.
\end{abstract}

\section{Educación a Distancia - Escuela Residencial - Estadísticas - Evaluación Formativa Tutores - Calidad}

\begin{abstract}
Research on residential schools has asked the summative question of whether schools are costeffective and also the formative question of how they might be improved. Greater statistical sophistication is required in distance education research. Statistical models of various aspects of residential schools are developed and tested. The main difference between student groups is in terms of the students' ratings of their group tutor's skills. A tutor's performance over the years remains at roughly the same level. A scale point difference in the rating of a tutor's skills is associated with half a scale point difference in the rating of the overall value of the school. The organisational impact of this work is noted.
\end{abstract}




\section{1. ¿QUÉ HAY QUE HACER CON LAS ESCUELAS RESIDENCIALES?}

En diversas partes del mundo, las escuelas residenciales han venido siendo "una parte integrante de la provisión de educación a distancia" (Eiseman, 1992). Los educadores a distancia han estado siempre preocupados por las críticas referentes a la inferioridad de la educación a distancia con respecto a la educación tradicional en el aula y siempre han estado buscando la manera de superar esta supuesta deficiencia de no tener ninguna interacción en el aula. Por supuesto, la solución más obvia para los educadores a distancia es comprometer y complementar los métodos a distancia con algunos elementos de la tutoría presencial (Burt, 1997, pp. 99-109). Pero, ¿cómo organizar esta tutoría presencial? Para periodos relativamente cortos de tutoría los estudiantes pueden desplazarse de sus hogares a los lugares de la tutoría para asistir a las mismas y volver a casa en el mismo día. Para periodos más largos el estudiante debe pernoctar una o varias noches en el lugar de tutoría: este tipo de lugares de tutoría es conocido como escuela residencial.

Sin embargo, como veremos, el papel de estas escuelas residenciales ha sido objeto de muchas discusiones. Para encontrar la respuesta a la pregunta de "¿qué hay que hacer con las escuelas residenciales?”, resulta útil realizar tres distinciones. Una forma de contestar a esta pregunta sería llevar a cabo un proceso de razonamiento y deducir cuál sería el mejor curso de acción. Aquí la pregunta se interpreta como una invitación al análisis racional. Alternativamente, la pregunta puede interpretarse como una invitación al análisis social/político en el ámbito organizativo o social. De esta forma, McIntosh (1975) señala que "existen muchísimas personas con diferentes intereses particulares" en el tema de las escuelas residenciales. Lo que suceda con ellas dependerá del juego mutuo entre esos diversos intereses particulares. Existe una segunda distinción totalmente independiente, que refleja la distinción usual entre evaluación sumativa y formativa. Aquí la pregunta sumativa es “'hay que dotar de escuelas residenciales?” y la pregunta formativa es “¿cómo podemos mejorar las escuelas residenciales?”. Una distinción final se refiere a la metodología de investigación que se debe emplear en cualquier investigación sobre las escuelas residenciales. Una distinción habitual en la literatura es la que se efectúa entre los enfoques cualitativos y cuantitativos. Una distinción menos habitual es la que existe entre el uso de estadísticas de una forma descriptiva y el uso de estadísticas de una forma más sofisticada, en orden a sacar deducciones. De acuerdo con este capítulo introductorio, la parte fundamental del presente informe da cuenta de una evaluación formativa orientada a la mejora de las escuelas residenciales, utilizando el análisis racional y unas estadísticas deductivas sofisticadas.

En primer lugar, examinemos la pregunta sumativa "¿hay que dotar de escuelas residenciales?”. Para responderla, hemos de tomar en consideración la rentabilidad. Durante las últimas décadas se ha producido una dramática expan- 
sión mundial de la educación a distancia. Esta expansión se ha debido en gran medida a la presión para incrementar el número de estudiantes en proceso educativo, unido a la incapacidad o a la falta de voluntad de dotar de los recursos que permitirían a los alumnos recibir la enseñanza en el campus. Rumble (1997, p. $161,204)$ observa que "existen numerosas pruebas de que la enseñanza abierta y a distancia pueden ser más rentable que las formas tradicionales de enseñanza...", pero advierte que "este no es necesariamente el caso" y cita la conclusión más bien cautelosa de Perraton (1982, p. 61) de que "existen circunstancias en que la educación a distancia parece atractiva desde un punto de vista económico". La mayor rentabilidad de la educación a distancia requiere el recorte de la provisión de medios de enseñanza que suponen un coste elevado por alumno. Sin embargo, este argumento económico a menudo entra en conflicto con un argumento educativo que apunta al hecho de que los medios (M) contribuyen a alcanzar algún fin educativo deseable (E):

La educación convencional es buena porque proporciona medios (M).

Los medios (M) son buenos porque proporcionan el fin (E).

La educación a distancia debe proporcionar medios $(M)$.

Podemos encontrar este argumento general aplicado a las escuelas residenciales. Se piensa que las escuelas residenciales sirven a una variedad de fines educativos deseables. Refiriéndose a las instituciones duales, Brackenreg y Allan (1991) enumeran las siguientes funciones posibles: identificación con el campus en el que los alumnos van a graduarse, acceso a los recursos del campus, relaciones con otros alumnos, aumento de la autoestima a través de la interacción con sus iguales, acceso a un "rito de paso", identificación con la cultura estudiantil, el efecto "refugio", motivación para continuar, desarrollo de técnicas orales, desarrollo de una mayor responsabilidad y relación con los alumnos por parte del profesor, aprendizaje del "sistema" y clarificación académica del material de enseñanza. En una discusión amplia sobre las escuelas residenciales, Morgan y Thorpe (1993) identifican las siguientes justificaciones fundamentales de las escuelas residenciales: transformación de la comprensión del alumno, acceso a formas particulares de experiencia de aprendizaje y acceso a una enseñanza especializada.

Algunos autores contrarrestan estas justificaciones argumentando que los costes para los alumnos y para las instituciones son excesivamente elevados. Las escuelas residenciales son caras por dos motivos: en primer lugar, implican una enseñanza presencial (Rumble, 1997) y, en segundo lugar, exigen viajar y quedarse a dormir lejos del hogar (Hermann et al., 1991). En términos de coste institucional, Brackenberg and Allan (1991, p. 29) resñala que "en el actual clima de racionalización económica existe una tendencia a... suprimir la escuela residencial como componente obligatorio de un curso". Sobre el coste para el alumno, Cuskelly y Dekkers (1992, p. 136) sugieren que "los costes para los alumnos asociados a las actividades en el campus no son desdeñables y pueden muy bien resul- 
tar un factor de disuasión para que los potenciales alumnos se inscriban en [tales] cursos". Ver también Parer (1988). Una investigación importante sobre los costes y beneficios de las actividades en el campus es la de Cameron et al. (1991).

Los críticos continúan argumentando que pueden alcanzarse los mismos resultados educativos por otros medios. Eiseman (1992) señala que las escuelas residenciales han formado "parte integrante de la provisión para los estudios a distancia en Australia", pero arguye que deben "contemplarse [únicamente] como una estrategia para alcanzar los resultados educativos perseguidos". Señala que "los alumnos discuten cada vez más la necesidad de actividades obligatorias en el campus y piden alternativas". Eiseman y Mahoney (1992) dan cuenta de una evaluación a pequeña escala de tal alternativa. Kember (1982, p.224) señala que la escuela residencial ha constituido a menudo el medio preferido de llevar a cabo trabajos prácticos, pero se pregunta también "si se han investigado por completo las alternativas y si se han tenido debidamente en cuenta la eficacia educativa y la conveniencia para el alumno".

A la vista de estos debates, no resulta sorprendente que no todas las instituciones de educación a distancia ofrezcan escuelas residenciales. (Rekkedal et al. (1989) descubrieron que el 88\% de las treinta y dos instituciones de correspondencia examinadas utilizaban enseñanza presencial de alguna clase, de forma que el porcentaje de utilización de las escuelas residenciales debería ser del 88\% o inferior). Incluso una institución como la Open University del Reino Unido, que ofrece algunas escuelas residenciales, solamente lo hace para una minoría de sus cursos. En determinados cursos en los que tradicionalmente se ofrecían escuelas residenciales se está retirando este componente. Solamente el 30\% de los cursos para no graduados ofrecidos en 1996 tenía escuelas residenciales. En contraste con otras facultades, la mayor parte $(65 \%)$ de los cursos de Ciencias contaba con escuelas residenciales. Fuera del programa para no graduados, la Escuela de Negocios de la Open University tenía una proporción similar de cursos con escuelas residenciales.

Por otra parte, cuando se ofrecen escuelas residenciales, los alumnos dicen que los encuentran útiles. Por ejemplo, Siaciwena (1990) informa de las elevadas tasas de utilidad de las escuelas residenciales en la Universidad de Zambia. Una prueba que exige más esfuerzo en relación con las escuelas residenciales es la de compararlas con otros componentes educativos. Timmins (1989) descubrió que las escuelas residenciales aparecen en tercer lugar, detrás de las guías de estudio y de los libros de texto. Los estudios de los componentes educativos efectuados en la Open University del Reino Unido revelaron que las escuelas residenciales figuraban como el componente educativo más útil en 1972, por delante de la enseñanza por correspondencia, la televisión, las tutorías, el asesoramiento y la radio (McIntosh, 1975, p. 59). Estudios más recientes mostraron que las escuelas residenciales aparecían como: el tercer componente educativo más útil (de entre 
Calidad de los Tutores en las Escuelas Residenciales: un Análisis Estadístico

catorce componentes educativos) durante el período de 1984-1990; el segundo componente educativo más útil (de entre veintiséis componentes educativos) en el año 1991; y el quinto componente educativo más útil (de entre veintiséis componentes educativos) en el año 1995 y también en el año 1996. Cuando no existen escuelas residenciales, los alumnos creen que sus estudios se habrían visto beneficiados en alguna medida de haber contado con escuelas residenciales. (Burt, 1997, pp. 10, 18, 117).

Los resultados a que nos hemos referido en el párrafo anterior son discutibles. En primer lugar, están basados en las opiniones de los alumnos, mientras que nuestra primera preocupación debería referirse a lo que ha aprendido el alumno. En segundo lugar, lo que se recoge en el párrafo anterior son estadísticas descriptivas. No existe la intención de elaborar un modelo estadístico y sacar deducciones estadísticas. En el que constituye uno de los estudios más impresionantes sobre cualquier aspecto de la educación a distancia, elaborado en la Open University del Reino Unido (y desgraciadamente aún sin publicar), dos de mis colegas, Gillie y Woodley (1995) remedian estos dos defectos. Llevan a cabo un análisis estadístico de los efectos de la asistencia a escuelas residenciales sobre los rendimientos comprobados de los alumnos. Su conclusión es que la asistencia a escuelas residenciales añadió un 1\%,2\%, 4\% y $6 \%$ a las calificaciones en los exámenes durante los cinco cursos en que investigaron. Contrastaron estos resultados con las percepciones que tenía el personal académico en relación con los efectos de las escuelas residenciales. El personal académico pensaba que la escuela residencial añadía un 10\%, 9\%,7\%,9\% y 7\% a las calificaciones de los exámenes durante los cinco cursos. El personal académico sobrevaloraba claramente los efectos de la escuela residencial. Gillie y Woodley ofrecen también cálculos de los costes de las escuelas residenciales -tanto para la universidad como para los alumnos- y contrastan el tamaño de los costes con el de los efectos.

Regresamos ahora a la cuestión formativa de cómo mejorar las escuelas residenciales. La mejora cualitativa es posible, tanto en términos de componentes sustantivos del colegio como en términos de la formación de los tutores empleados. Las decisiones relativas a la mejora de la calidad pueden inspirarse en la evaluación. Se pueden obtener las respuestas de los alumnos frente a componentes específicos del colegio (por ejemplo Warner y Wilkinson, 1992). Se pueden obtener también las respuestas de los alumnos frente a los tutores. Morgan y Thorpe (1993, pp. 79-80) señalan que "un informe reciente sobre la calidad de la enseñanza en la Open University del Reino Unido puso de manifiesto la necesidad de un mayor desarrollo del personal para mejorar la enseñanza en las escuelas residenciales".

No obstante, los resultados de muchas evaluaciones formativas de las escuelas residenciales pueden discutirse, de la misma manera que pueden discutirse las evaluaciones sumativas. No solamente porque están basadas en las opiniones 
de los alumnos, sino también porque contienen a menudo estadísticas descriptivas. No existe la intención de elaborar un modelo estadístico y realizar deducciones estadísticas. La finalidad del presente informe es mostrar cómo pueden remediarse estos defectos. Se elaboran modelos estadísticos para distintos aspectos de las escuelas residenciales y se comprueban. Se sacan deducciones. Aunque el estudio hace uso de datos de opinión, dichos datos no se toman al pie de la letra -sino que su fiabilidad y validez se cuestiona continuamente por medio de diversos análisis estadísticos.

\section{LA EVALUACIÓN FORMATIVA DE UNA ESCUELA RESIDENCIAL}

Para informar el proceso de mejora de la calidad, la Open University del Reino Unido lleva a cabo unas evaluaciones periódicas de las escuelas residenciales. Aquí utilizaré los datos procedentes de esas investigaciones para tratar las tres cuestiones siguientes:

¿Poseen las escuelas residenciales una calidad elevada?

¿Existe una uniformidad de resultados a lo largo de los lugares, años y cursos?

¿Es válida y fiable la respuesta frente a los tutores?

En la Parte 1 comienzo señalando la necesidad del modelo que constituye la base del análisis. Examino después la calidad y la uniformidad del producto. En la Parte 2 examino la respuesta frente a los tutores en su actuación.

Estas cuestiones se investigan en relación con la escuela residencial durante un curso en particular, a saber, el de "Gestión de empresas benéficas y sin fines de lucro" (código del curso: B789). Este curso forma parte del Programa de Gestión de las Organizaciones Benéficas de la Open Business School. La escuela residencial funcionó en la primavera de 1997, hacia el final del estudio del curso por los alumnos. Es un curso en el cual he venido realizando evaluaciones sobre las escuelas residenciales dos veces al año, desde el año de 1992. Ya desde las primeras evaluaciones se extraen algunas conclusiones, así como de mis evaluaciones paralelas de la escuela residencial del B974, que es un curso hermano del B789 cuya escuela residencial tiene un formato similar. El calendario de la escuela residencial del B789 figura más abajo. El colegio funciona desde las últimas horas de la tarde del Día 1hasta media tarde del Día 3. Existen ocho sesiones, que tratan diversos temas: una sesión en la tarde del Día 1, cuatro sesiones en el Día 2 y tres sesiones en el día 3. 
Calidad de los Tutores en las Escuelas Residenciales: un Análisis Estadístico

Tabla 1: Horario de la escuela residencial del B789

\section{Día 1}

\begin{tabular}{ll} 
Desde las $4.40 \mathrm{pm}$ & Llegada e inscripción \\
\hline Sesión preliminar & Bienvenida al curso y reunión informativa \\
\hline Sesión 1 & Presentación del grupo. Estudio del caso práctico Jan Morrow \\
\hline
\end{tabular}

\section{Día 2}

\begin{tabular}{ll} 
Sesión 2 & Haciendo inventario \\
\hline Sesión 3 & Seminario de Evaluación o Negociación \\
\hline Sesión $\mathbf{4}$ & Seminario de Negociación o Evaluación. Tiempo libre \\
\hline Sesión 5 & Gestión en la práctica \\
\hline
\end{tabular}

\section{Día 3}

\begin{tabular}{ll} 
Sesión 6 & Gestión de valores y cambio \\
\hline Sesión 7 & Gestión de valores y cambio (continuación) \\
\hline Sesión 8 & Sesión de recapitulación. Sesión de información sobre el examen \\
\hline Final & $3.00 \mathrm{pm}$ \\
\hline
\end{tabular}

\section{CALIDAD Y UNIFORMIDAD DE LA DOTACIÓN (Parte 1)}

\subsection{UN MODELO DE ESCUELA RESIDENCIAL}

El fundamento que subyace en mi análisis es el de considerar la escuela residencial como un sistema. Se efectúan unas entradas en el sistema y el sistema produce unos resultados.

La primera de nuestras tres preguntas se relaciona con la calidad de las entradas y de los resultados. La segunda y la tercera se refieren ambas a cómo se relacionan las entradas con los resultados. La segunda se pregunta si diferentes "entradas" de localizaciones, años y cursos producen resultados uniformes o diferentes. La tercera pregunta se refiere a cuál es el efecto de diferentes "entradas" de tutores.

Figura 1. Un modelo input-output 
Las entradas son diversas. Una manera útil de visualizar estas entradas es imaginar que se dirige una nota a un alumno de la escuela residencial. Al alumno se le incluye en un grupo, bajo la dirección de un tutor determinado. En todo momento, el alumno estará en una de las sesiones dirigidas por uno de los tutores. Estas tiene lugar en una de las escuelas dirigidas por un director de curso, en la escuela residencial del B789 en la primavera de 1997, ya sea en el Reino Unido o en Zimbabwe.

Figura 2. "Dirección" de los inputs

\begin{tabular}{l}
\hline alumno \\
\hline grupo de alumnos \\
\hline tutor del grupo de alumnos \\
\hline sesión \\
\hline grupo de la sesión \\
\hline tutor del grupo de la sesión \\
\hline escuela (localización y semana) \\
\hline director de la escuela \\
\hline país \\
\hline presentación \\
\hline año \\
\hline diseño de la escuela residencial \\
\hline curso
\end{tabular}

En este estudio los resultados son las calificaciones que dan los alumnos a diversos aspectos de la escuela residencial. Se pide al alumno que califique cada uno de los cursos en términos de "interés y diversión", "importancia del curso" y "utilidad para él personalmente". También combino estas tres calificaciones para calcular una calificación compuesta de cada curso. El curso de Preparación para el Examen se califica en términos de "presentación y diversión" y "utilidad". Cada grupo de alumnos califica a sus tutores respecto de cinco capacidades: explicación del trabajo; facilitación del debate; responsabilidad frente a los individuos y apoyo de los mismos; explicación de ideas y temas; y conocimiento de "lo que sucede" en las organizaciones benéficas y sin fines de lucro. Aquí combino también estas calificaciones para calcular una calificación compuesta, esta vez la de la capacidad del tutor. El director del curso se califica en función de su disponibilidad y utilidad. El funcionamiento de la escuela se califica en cuanto a documentos, alojamiento y comida, organización de las opciones y administración. Los alumnos califican también la escuela en relación con si es "amigable, favorable al estudio y no discriminatoria”. Finalmente, los alumnos dan una evaluación general relativa a en qué medida encuentran útil la escuela residencial. 
Calidad de los Tutores en las Escuelas Residenciales: un Análisis Estadístico

Tabla 2. Outputs

\begin{tabular}{|c|c|}
\hline Sesión: & $\begin{array}{l}\text { Interés y diversión; importancia para el curso; } \\
\text { utilidad personal; conjunto }\end{array}$ \\
\hline Preparación del examen: & presentación y diversión; utilidad \\
\hline Tutor: & $\begin{array}{l}\text { Explicación del trabajo, facilitación del debate, responsabilidad y } \\
\text { apoyo de las personas; explicación de ideas y temas; } \\
\text { conocimiento de "lo que pasa" } \\
\text { organizaciones benéficas y sin fines de lucro; conjunto }\end{array}$ \\
\hline Director del curso: & Disponibilidad; utilidad \\
\hline Escuela: & $\begin{array}{l}\text { Documentación; alojamiento y comida; opciones administración; } \\
\text { amigable, favorable al estudio y no discriminatoria }\end{array}$ \\
\hline Global: & Utilidad \\
\hline
\end{tabular}

Lo que queremos saber es: ¿cuál es el efecto de cada entrada? En relación con cada resultado, queremos saber cuáles entradas son las más importantes para determinar tal resultado. En relación con cada entrada, queremos saber los resultados sobre los que más influye.

Parece bastante natural esperar lo siguiente:

i diferentes tutores y diferentes grupos de alumnos pueden dar lugar a diferentes calificaciones de la capacidad del tutor y diferentes calificaciones de las sesiones del grupo;

ii diferentes localizaciones de la escuela y diferentes directores del curso pueden dar lugar a diferentes calificaciones del funcionamiento de la escuela y de la sesión de Preparación del Examen (normalmente presentada a todos los alumnos juntos, ya sea por uno de los tutores o por el director del curso);

iii diferentes países pueden dar lugar a diferentes calificaciones de aspectos relacionados con la importancia del contexto cultural de los alumnos.

Para ilustrar mi método, examinemos el efecto que tienen las distintas escuelas sobre la utilidad de la sesión de Preparación del Examen. La primera fila de la Tabla 3 que figura más abajo muestra las principales calificaciones de la utilidad del curso de Preparación del Examen en cada una de las cuatro escuelas del Reino Unido. Las cuatro escuelas están localizadas en cuatro lugares distintos: Cookham, Instituto de Ciencia y Tecnología de la Universidad de Man- 
chester (UMIST), Livingston Hilton y Harben House. Cookham fue calificada con un 4.5, etc. Seleccionamos ahora una de las cuatro escuelas para que sirva de punto de referencia. Es un poco arbitrario -aquí el ordenador toma Harben House como punto de referencia. La segunda fila de la tabla proporciona las calificaciones medias en relación con la media de Harben House. Por ejemplo, la calificación media de Cookham en relación con la de Harben House es de 4.5$3.7=0.8$. Decimos que el alcance del efecto de la escuela en Cookham es de 0.8. De esta forma, existe casi un punto entero de diferencia en la escala entre Cookham y Harben House.

Sin embargo, tenemos que comprobar que esta diferencia no se debe únicamente a una variación debida a la casualidad. Las pruebas del ordenador descubren que no se debe únicamente a la casualidad, en otras palabras, es "estadísticamente significativa". He indicado este extremo mediante un *. El efecto UMIST también es de un 0.8 y también es significativo. No obstante, el efecto de la escuela Livingston es de solo un 0.3 y no resulta significativo, es decir, no es significativamente diferente del de Harven House.

Tabla 3. El efecto de las diferentes escuelas sobre la utilidad de la Preparación del Examen

\begin{tabular}{|l|c|c|c|c|}
\hline & Cookham & UMIST & livingston & $\begin{array}{c}\text { Harben House } \\
\text { (referencia) }\end{array}$ \\
\hline $\begin{array}{l}\text { Utilidad de la Preparación del Examen } \\
\text { calificaciones medias }\end{array}$ & 4.5 & 4.5 & 4.0 & 3.7 \\
\hline $\begin{array}{l}\text { Media en relación con la referencia } \\
\text { [magnitud del efecto] }\end{array}$ & $.8^{*}$ & $.8^{*}$ & .3 & 0 \\
\hline
\end{tabular}

Nota: Las diferencias significativas se indican *, $\mathrm{p}<0.05$.

\section{2. ¿POSEEN UNA CALIDAD ELEVADA LAS ESCUELAS RESIDENCIALES?}

Existen buenos motivos para suponer que las entradas son de elevada calidad. Las calificaciones de los alumnos demuestran que la calidad de los resultados es muy elevada.

El diseño de la escuela residencial por el equipo de estudios de la sede central de la Open University en Walton Hall es el resultado de un esfuerzo intensivo, tanto en la fase inicial de diseño como en las posteriores de revisión del mismo. Los tutores se reclutan a través de un proceso de selección y muchos de ellos han tenido experiencia como tutores en la escuela residencial con anterioridad y están familiarizados con el curso, ya sea a través de la pertenencia al equipo del 
curso o por haber sido tutores del curso. Los alumnos acuden también a la escuela residencial después de haber trabajado con los materiales durante la primera mitad del curso. Finalmente, las localizaciones han sido seleccionadas por su adecuación.

Los 276 alumnos de la escuela residencial del B789 correspondiente a la primavera de 1997 calificaron cada pregunta de 1 (mínimo) a 5 (máximo). Las calificaciones medias de la Tabla 4 se encuentran casi todas entre el 4 y el 5, es decir, casi en la cima de la escala. La valoración general de la escuela residencial es de 4.4.

Tabla 4. Elevada calidad de los resultados:

calificaciones medias de la escuela residencial del B789, primavera 1997

\begin{tabular}{llc} 
Sesión 1: & Presentaciones y estudio caso práctico Jan Morrow & calfificación media \\
& interesante y divertido & \\
& importante para el curso & 4.0 \\
& útil para mí personalmente & 4.4 \\
Sesión 2: & Haciendo balance & 3.9 \\
& interesante y divertido & \\
& importante para el curso & 4.1 \\
& útil para mí personalmente & 3.7 \\
Sesiones 3/4: & Seminario de negociación & 3.8 \\
& interesante y divertido & \\
& importante para el curso & 3.9 \\
& útil para mí personalmente & 4.4 \\
\hline
\end{tabular}

Sesión 5: $\quad$ Gestión en la práctica

interesante y divertido

importante para el curso

4.1

útil para mí personalmente

3.9

\section{Sesiones 6 y 7: Gestión de Valores y Cambio}

interesante y divertido

4.2

importante para el curso

4.4

útil para mí personalmente

4.3

\section{El tutor de su grupo nuclear*}

explicación de lo que debíamos hacer

responsabilidad/apoyo de las personas 


\section{Sesión 8: $\quad$ Preparación del Examen}

bien presentado y manejado $\quad 4.0$

útil $\quad 4.2$

\section{Funcionamiento de la escuela*}

documentos

4.0

alojamiento y comida

4.3

administración

4. 1

\section{Director del curso}

$\begin{array}{ll}\text { disponible y accesible } & 4.1 \\ \text { capaz de ayudar y responder a preguntas } & 3.9\end{array}$

\section{Valoración global de la escuela**}

Nota: en la mayoría de las escalas, " 5 " se calificaba sencillamente por ejemplo "interesante y divertido", mientras que "1" se calificaba "no interesante ni divertido"; en el caso de *, $5=$ excelente y 1 = pobre; en el caso de ${ }^{* *}, 5=$ muy útil; 1 = nada útil

\subsection{UNIFORMIDAD DE LOS RESULTADOS A LO LARGO DE LOS CURSOS Y DE LOS AÑOS}

La escuela residencial del curso B789 ha mantenido una elevada calificación de manera uniforme desde que se celebró la primera en 1992 -al igual que su curso hermano, el B794.

Las calificaciones están todas en una franja entre 4.1 y 4.4 .

Tabla 5. Uniformidad a lo largo de años y cursos

\begin{tabular}{|c|c|c|c|c|c|c|c|c|c|c|}
\hline $\begin{array}{c}\text { Año } \\
\text { y presentación }\end{array}$ & $92 \mathrm{P}$ & 920 & 93P & 930 & 94P... & $95 \mathrm{P}$ & 950 & 96P & 960 & $97 \mathrm{P}$ \\
\hline \multicolumn{11}{|c|}{ Valoración global de la escuela residencial } \\
\hline B789 & 4.3 & 4.2 & 4.3 & 4.3 & $4.3 \ldots$ & 4.2 & 4.3 & 4.1 & $\ldots$ & 4.4 \\
\hline B794 & & & & 4.2 & $4.3 \ldots$ & $\ldots$ & $\ldots$ & 4.4 & 4.4 & 4.3 \\
\hline
\end{tabular}

Notas:

(i) 5 = muy útil; 1 = nada útil;

(ii) Hay dos presentaciones cada año, una que va del otoño a la primavera y otra que va del verano al otoño. $\mathrm{P}=$ escuela de primavera; $\mathrm{O}=$ escuela de otoño 


\subsection{UNIFORMIDAD DEL RESULTADO SEGÚN LOS PAÍSES}

En la primavera de 1997 la escuela residencial B789 fue ofrecida en cuatro lugares del Reino Unido y también en Harare y Zimbabwe.

En la mayor parte de las magnitudes no existen diferencias significativas entre el Reino Unido y Zimbabwe. Las escasas diferencias que podemos detectar insinúan algunas diferencias culturales.

La Tabla 6 muestra el alcance del efecto debido al país, tomando Zimbabwe como punto de referencia. Las diferencias fueron de alrededor de un tercio de punto de la escala. Zimbabwe tenía calificaciones más bajas: en cuanto al interés y diversión, en Haciendo Inventario; en cuanto a la utilidad personal, en Gestión de Valores y Cambio; y por su tutor principal en "Conocer lo que sucede en las organizaciones benéficas y sin fines de lucro”. ¿Pudiera haberse debido a que los tutores de Harare (cuatro de los cinco tutores eran ciudadanos del Reino Unido) tuviesen dificultades para referir "lo que sucede" en las organizaciones benéficas y sin fines de lucro en Zimbabwe? Zimbabwe tuvo calificaciones más altas en el interés y la utilidad del Taller de Negociación. ¿Son, por algún motivo, más importantes estos temas en el contexto de Zimbabwe?

Tabla 6. Efectos en los dos diferentes países

\begin{tabular}{lll} 
& Reino Unido & Zimbabwe \\
Sesión: Interés de "Haciendo balance" & $+.4^{*}$ & 0 \\
\hline Sesión: Interés de "Evaluación" & $-.3^{*}$ & 0 \\
\hline Sesión: Utilidad de "Evaluación" & $-.4^{*}$ & 0 \\
\hline Sesión: Utilidad de "Negociación" & $-3^{*}$ & 0 \\
\hline Sesión: Utilidad de "Valores" & $+.4^{*}$ & 0 \\
\hline Tutor: Conocimiento de "lo que pasa" & $+.5^{*}$ & 0 \\
\hline
\end{tabular}

Nota: Las diferencias significativas se indican con ${ }^{*}, \mathrm{p}<0.05$

\subsection{UNIFORMIDAD DE LOS RESULTADOS ENTRE LAS ESCUELAS}

En la mayor parte de las magnitudes no existen diferencias significativas entre las diferentes escuelas del Reino Unido. Los únicos efectos de la escuela se encuentran en el alojamiento y la comida, en la sesión de preparación del examen y en la capacidad de un tutor determinado. 
La Tabla 7 muestra el alcance del efecto debido a la escuela, tomando Harben House como punto de referencia. Las diferencias alcanzaron en algunos casos hasta un punto en la escala. Había cuatro escuelas en el Reino Unido: Cookham, UMIST, Livingston Hilton y Harben House. Por lo que se refiere al funcionamiento de la escuela, la única diferencia afectó al alojamiento y la comida: Cookham y UMIST recibieron una calificación más elevada que Harben House, que recibió a su vez una calificación más elevada que Livingston Hilton. No existieron diferencias significativas en las calificaciones del director de curso. En términos de la sesión de Preparación del Examen, Cookham y UMIST recibieron una calificación superior a las de Livingston Hilton y Harben House.

Es menos probable que existan diferencias en las calificaciones de las escuelas relativas a los tutores, porque la mayor parte de las escuelas tuvieron cuatro o seis tutores y, así, cualquier diferencia entre los mismos tendería a prorratearse. No obstante, Cookham y Livingston consiguieron mejores calificaciones de sus tutores en la explicación de ideas o temas de gestión difíciles. Livingston consiguió mejores calificaciones por sus tutores en el apartado de conocer "lo que pasa" en las organizaciones voluntarias y sin fines de lucro.

Tabla 7. Efectos de diferentes escuelas

\begin{tabular}{|l|c|c|c|c|}
\hline & Cookham & \multicolumn{1}{c}{ UMIST } & livingston & Harben House \\
\hline escuela: alojamiento & $.4^{*}$ & $.4^{*}$ & $-.3^{*}$ & 0 \\
\hline sesión: presentación prep. examen & $1.0^{*}$ & $1.3^{*}$ & $.5^{*}$ & 0 \\
\hline sesión: ayuda preparación examen & $.7^{*}$ & $.8^{*}$ & .3 & 0 \\
\hline tułor: explicación del trabajo & $.5^{*}$ & 0 & $.4^{*}$ & 0 \\
\hline tułor: explicación de ideas & .4 & -.1 & $.4^{*}$ & 0 \\
\hline tutor: conoce... & .2 & -.1 & $.5^{*}$ & 0 \\
\hline
\end{tabular}

Nota: Las diferencias significativas se indican con ${ }^{*}, \mathrm{p}<0.05$

\section{IMPRESIONES SOBRE LA ACTUACIÓN DE LOS TUTORES (Parte 2)}

Durante muchos años la Open University ha solicitado a los alumnos de las escuelas residenciales que comenten la actuación de los tutores de su escuela residencial. Estos comentarios se entregan a su vez a los tutores, de forma que éstos puedan medir la calidad de su actuación y, en su caso, mejorarla. La información puede ser utilizada también por el equipo del curso en decisiones relacionadas con los tutores, incluida la selección de tutores para futuras escuelas residenciales. 
Calidad de los Tutores en las Escuelas Residenciales: un Análisis Estadístico

Los comentarios de los alumnos acerca de los tutores de su escuela residencial son importantes para dichos tutores, para el equipo del curso y para futuros alumnos. ¿Qué fiabilidad y validez tiene esta información?

Cada año, desde 1992, he llevado a cabo dos evaluaciones anuales de la escuela residencial del curso B789 Gestión de empresas benéficas y sin fines de lucro. A lo largo de este período he subrayado en mis impresiones sobre los tutores que la información debía utilizarse con cautela. Esto se ilustra en el resumen de una hoja de impresiones típica que figura a continuación.

\section{RESPUESTAS SOBRE LA ACTUACIÓN DEL TUTOR EN LA ESCUELA RESIDENCIAL DEL B789, primavera de 1996}

A continuación aparecen las respuestas a diversos indicadores relacionados con la escuela residencial. Las cifras de la columna de la izquierda dan la respuesta media de todos los alumnos y las cifras de la columna del medio dan la respuesta media de sus propios alumnos. La columna de la derecha da el error estándar de su media. Su media "real" es probable que tenga un recorrido de dos errores estándar de la cifra dada. También figura el número de alumnos de su grupo particular que han respondido al cuestionario.

Hay que adoptar diversas precauciones a la hora de interpretar estas cifras. En primer lugar, el número de alumnos es bajo, a veces muy bajo; los que contestan pueden no ser representativos del conjunto del grupo de Vd.; el grupo de Vd. puede ser más - menos- generoso que otros grupos; su propia actuación como tutor puede cambiar a lo largo del tiempo; y puede cuestionarse algo la fiabilidad y validez de los indicadores utilizados (aunque la pasada experiencia con indicadores similares sugiere al menos cierta fiabilidad y validez). Así que, con todas estas advertencias, iaqui están los resultados!. Espero que encuentren de utilidad la información.

Tutor: Jean Smith

Número de alumnos de su grupo que han respondido: 13

\begin{tabular}{|l|c|c|c|}
\hline Explicar el trabajo a realizar & $\begin{array}{c}\text { Todos } \\
\text { los alumnos }\end{array}$ & $\begin{array}{c}\text { Su } \\
\text { media }\end{array}$ & $\begin{array}{c}\text { Error } \\
\text { estóndar }\end{array}$ \\
\hline Facilitar el debate & 4.3 & 4.9 & 0.1 \\
\hline Responder a las personas & 4.2 & 4.9 & 0.1 \\
\hline Explicar ideas y temas & 4.3 & 5.0 & 0.0 \\
\hline Saber lo que pasa en las organizaciones & 4.2 & 4.8 & 0.2 \\
\hline
\end{tabular}

NB: $5=$ Excelente... $1=$ Pobre 
Aquí la cuestión fundamental es:

¿Tienen distintos tutores distintos efectos?

No obstante, para separar la influencia del tutor de la influencia de otros aspectos de la escuela residencial, también preguntamos:

¿Permanecen iguales a lo largo de los años las diferencias entre tutores?

Finalmente, preguntamos:

¿La capacidad del tutor tiene una influencia en la valoración general del alumno acerca de la escuela?

\section{1. ¿CÓMO DIFIEREN LOS GRUPOS DE ALUMNOS EN LAS CALIFICACIONES QUE OTORGAN? ¿TIENEN DISTINTOS RESULTADOS LOS DISTINTOS TUTORES?}

Obsérvese que en este estadio del análisis todavía no somos capaces de separar las respuestas a estas dos preguntas.

Cada escuela tenía entre cuatro y seis grupos de alumnos, con unos once alumnos cada uno. En total había veinticinco grupos de alumnos ("grupos nucleares"). Cada grupo de alumnos tenía su propio tutor ("tutor del grupo nuclear").

La principal diferencia entre los tutores se produce en términos de las calificaciones de los alumnos relativas a la capacidad del tutor de su grupo nuclear. Los tutores parecían influir también en la valoración general. Parecían igualmente influir en las calificaciones de determinados cursos, pero no de otros.

[Existen también variaciones entre los distintos grupos de alumnos en sus calificaciones relativas al funcionamiento de la escuela y al curso de Preparación del Examen -pero esta variación deja de ser significativa una vez que se ha tenido en cuenta la influencia de la escuela.]

¿Por qué existen variaciones entre los distintos grupos de alumnos de algunas sesiones pero no de otras? Dos de las sesiones en que existen variaciones según el grupo son la sesión de Introducción (y el estudio del caso práctico Jan Morrow) y la sesión de Gestión de Valores y Cambio. Estas dos sesiones pueden depender más del interés del tutor que otras asignaturas. Otra explicación podría ser que estos dos cursos son el primero y el último, de forma que pueden haber causado una mayor impresión en las mentes de los alumnos. Otra consideración es que Gestión de Valores ocupaba dos sesiones, en lugar de una. 
Otras dos sesiones en las que se producen variaciones en función del grupo son el seminario de Evaluación y el seminario de Negociación. Estos fueron los dos cursos en que (habitualmente) los alumnos fueron trasladados de su tutor de grupo a otros tutores. En algunos casos los tutores dieron uno de los dos seminarios de Evaluación y Negociación. En otros casos, un tutor dio dos seminarios de Evaluación o dos seminarios de Negociación.

La Tabla 8 muestra la influencia de los tutores ( ¿o el efecto de los grupos de alumnos?) sobre seis magnitudes diferentes. Cinco de las magnitudes son puntuaciones compuestas obtenidas prorrateando varias de las calificaciones. La puntuación de Capacidad es la media de las cinco puntuaciones del tutor del grupo nuclear. La puntuación Valores es la media de las tres puntuaciones de la clase de Gestión de Valores y Cambio. Lo mismo sucede con las puntuaciones de Introducción, Evaluación y Negociación. La puntuación final es sencillamente la calificación de Evaluación Global de la escuela residencial.

El ordenador toma al tutor "James Bond" como punto de referencia. Su puntuación figura en la primera fila. Las puntuaciones restantes se otorgan en relación con la puntuación de James Bond. Un asterisco indica que la puntuación es significativamente diferente de la de James Bond desde el punto de vista estadístico. Existen muchas diferencias significativas entre los tutores individuales en el apartado Capacidad. En el caso de las demás variables, solo existen unas pocas diferencias significativas.

Consideraremos por separado cada columna de la tabla que figura a continuación. Los tutores A a J tienen unas calificaciones en Capacidad por encima de la media, los tutores $\mathrm{K}$ a $\mathrm{T}$ no difieren significativamente de la media, y los tutores U a Y tienen calificaciones en Capacidad por debajo de la media. Las calificaciones en Valores identifican solo a los tutores V y X como significativamente por debajo de la media. Las calificaciones en Introducción identifican solo a los tutores U e Y como significativamente por debajo de la media. Las calificaciones de Evaluación identifican solo a los tutores A, O, Q y X como significativamente por debajo de la media. Las calificaciones en Negociación identifican solo a los tutores E, U y W como significativamente por debajo de la media. (Sospecho sin embargo que las calificaciones de Evaluación y Negociación reflejan influencias del grupo nuclear, no influencias debidas a los tutores de Evaluación y Negociación). Las calificaciones de Evaluación General identifican solo a los tutores U, X e Y como significativamente por debajo de la media. 
Tabla 8. Calificaciones Tutor/Grupo de alumnos

\begin{tabular}{|c|c|c|c|c|c|c|}
\hline Tutor & Capacidad & Valores & Intro. & Eval. & Neg. & Global \\
\hline Bond & $4.1+$ & $4.4+$ & $4.1+$ & 4.4+ & $4.3+$ & $4.4+$ \\
\hline$A$ & $.8^{*}$ & .3 & .3 & 0 & -.2 & .2 \\
\hline$B$ & $.7^{*}$ & .3 & .4 & .1 & .3 & .3 \\
\hline C & $.7^{*}$ & .1 & .2 & -.3 & .1 & .2 \\
\hline$D$ & $.7^{*}$ & .2 & .1 & -.3 & $-.7^{*}$ & .0 \\
\hline$E$ & .7 & .2 & .2 & -.2 & 0 & .2 \\
\hline $\mathrm{F}$ & $.6^{*}$ & .2 & .3 & -.3 & 0 & .1 \\
\hline$G$ & .6 & .1 & .4 & -.5 & .1 & .2 \\
\hline $\mathrm{H}$ & $.6^{*}$ & .1 & .1 & -.2 & .4 & .3 \\
\hline I & $.5^{*}$ & .4 & .1 & -.3 & .2 & .3 \\
\hline$J$ & $.5^{*}$ & .0 & .3 & -.1 & .4 & .4 \\
\hline $\mathrm{K}$ & .5 & -.1 & -.1 & 0 & .2 & .3 \\
\hline $\mathrm{L}$ & .5 & .1 & .1 & $-.9 *$ & -.1 & .1 \\
\hline$M$ & .2 & -.2 & .1 & $-.8^{*}$ & -.1 & .0 \\
\hline $\mathrm{N}$ & .2 & -.2 & -.4 & .1 & -.1 & .1 \\
\hline 0 & .2 & -.1 & -.2 & -.3 & -.5 & -.1 \\
\hline$P$ & .1 & -.3 & .0 & -.1 & -.1 & -.3 \\
\hline$Q$ & .0 & .2 & .2 & -.2 & -.3 & .1 \\
\hline $\mathrm{R}$ & .0 & .0 & .0 & 0 & 0 & .0 [J. Bond] \\
\hline$S$ & .0 & .4 & .1 & -.4 & -.1 & -.1 \\
\hline$T$ & -.1 & -.2 & .0 & -.1 & -.2 & .0 \\
\hline U & $-.7^{*}$ & -.5 & $-.6^{*}$ & -.9 & $-.7^{*}$ & $-.8^{*}$ \\
\hline $\mathrm{V}$ & $-.7^{*}$ & $-.7^{*}$ & -.1 & -.2 & .2 & .3 \\
\hline W & $-8^{*}$ & -.5 & -.2 & .2 & -.2 & .0 \\
\hline$x$ & $-1.0^{*}$ & $-1.2^{*}$ & -.4 & $-1.1^{*}$ & -.2 & $-.9^{*}$ \\
\hline$Y$ & $-1.3^{*}$ & -.5 & $-.8^{*}$ & -.4 & $-1.1^{*}$ & $-.6^{*}$ \\
\hline
\end{tabular}

Nota: Las diferencias significativas se indican ${ }^{*}, p<0.05$. ¡Obsérvese que ${ }^{*}$ indica significativamente diferente de James Bond!

El análisis estadístico demuestra que existe una influencia significativa asociada al grupo de alumnos. Sin embargo, la calificación del alumno contiene también un error aleatorio. De esta forma, nuestra estimación de la influencia del grupo de alumnos contendrá un error aleatorio. Así podemos esperar que la media de un tutor varíe de una presentación a la siguiente. (Ver Apéndice 1). 


\section{2. ¿CONTINÚAN LAS DIFERENCIAS ENTRE TUTORES SIENDO LAS MISMAS A LO LARGO DE LOS AÑOS?}

La comparación de la actuación de un mismo tutor a lo largo de diferentes presentaciones de cursos indica un grado moderado de regularidad.

La Tabla 9 que figura a continuación muestra la calificación compuesta de la capacidad de los tutores correspondiente a cuatro presentaciones del B789: primavera de 1995, otoño de 1995, primavera de 1996 y primavera de 1997. Los tutores A, H, 2 y 4 están regularmente por debajo de la media. Sin embargo la calificación de cada uno de los tutores varía de unas presentaciones a otras y pueden existir discrepancias dramáticas -como por ejemplo en el caso del tutor G.

Tabla 9. Calificaciones Tutores/Grupos de alumnos

\begin{tabular}{|c|c|c|c|c|}
\hline \multirow[t]{2}{*}{ Tutor } & \multicolumn{4}{|c|}{ Calificaciones de capacidad } \\
\hline & P 1995 & 01995 & P 1996 & P 1997 \\
\hline$A$ & 4.9 & 4.7 & 4.9 & $4.9^{*}$ \\
\hline B & - & - & - & $4.8^{*}$ \\
\hline C & - & - & - & $4.8^{*}$ \\
\hline$D$ & - & - & {$[4.9]$} & $4.8^{*}$ \\
\hline$E$ & - & - & - & $4.8^{*}$ \\
\hline $\mathrm{F}$ & - & - & - & $4.7^{*}$ \\
\hline$G$ & - & - & 4.1,3.6 & $4.7^{*}$ \\
\hline $\mathrm{H}$ & 4.4 & 4.5 & 4.1 & $4.7^{*}$ \\
\hline I & - & - & - & $4.6^{*}$ \\
\hline$J$ & - & - & - & $4.6^{*}$ \\
\hline $\mathrm{K}$ & - & - & - & 4.6 \\
\hline $\mathrm{L}$ & 4.2 & - & 4.4 & 4.6 \\
\hline M & - & - & 4.4 & 4.3 \\
\hline $\mathrm{N}$ & - & - & - & 4.3 \\
\hline 0 & - & - & 4.5 & 4.3 \\
\hline$P$ & {$[4.2]$} & - & {$[4.4]$} & 4.2 \\
\hline$Q$ & 3.8 & - & - & 4.1 \\
\hline $\mathrm{R}$ & - & - & - & 4.1 \\
\hline$S$ & - & - & 4.3 & 4.1 \\
\hline$T$ & - & - & 4.3 & 4.0 \\
\hline U & - & - & 3.7 & 3.4 \\
\hline
\end{tabular}




\begin{tabular}{c|c|c|c|c}
\hline $\mathrm{V}$ & - & - & - & $3.4^{*}$ \\
\hline $\mathrm{W}$ & 3.9 & 2.5 & 4.1 & $3.3^{*}$ \\
\hline $\mathrm{X}$ & 3.3 & {$[2.5]$} & {$[4.1]$} & $3.1^{*}$ \\
\hline $\mathrm{Y}$ & - & - & - & $2.8^{*}$ \\
\hline 1 & 4.6 & - & - & - \\
\hline 2 & 4.5 & 4.3 & 4.3 & - \\
\hline 3 & 4.2 & - & - & - \\
\hline 4 & 4.2 & - & 4.5 & - \\
\hline 5 & - & 4.1 & - & - \\
\hline 6 & - & - & 4.1 & - \\
\hline 7 & 4.0 & - & - & - \\
\hline 8 & 3.8 & 4.0 & 3.9 & - \\
\hline 9 & 3.6 & - & - & - \\
\hline 10 & 3.6 & - & - & - \\
\hline 11 & - & 3.5 & 3.5 & - \\
\hline 12 & 3.3 & 3.3 & 3.5 & \\
\hline
\end{tabular}

Notas:

(i) Los datos correspondientes al otoño de 1995 están incompletos

(ii) Los tutores $\mathrm{A}$ a $\mathrm{Y}$ son aquellos que fueron tutores en primavera de 1997. Los tutores 1 a 12 son los que no lo fueron.

(iii) [4.7] indica que el tutor aparece en dos ocasiones en la lista de la A a la Y.

(iv) Las diferencias significativas se indican ${ }^{*}, \mathrm{p}<0.05$.

(v) Las seis correlaciones a la par entre las cuatro presentaciones son .7, .5, .5, .8*, 1.0* y .9*.

\subsection{UN ESTUDIO SIMILAR DE LA ESCUELA RESIDENCIAL DEL B794, CORRESPONDIENTE A LA PRIMAVERA DE 1997}

Un análisis similar de la escuela residencial del B794, correspondiente a la primavera de 1997, llegó a conclusiones similares en cuanto a la fuerte presencia de variaciones en las calificaciones del tutor del grupo nuclear según el grupo de alumnos.

Justo antes de mi estudio del B789, llevé a cabo un estudio similar en la escuela residencial del B794 correspondiente a la primavera de 1997. No me he preocupado de reflejarlo aquí con detalle, porque estaba basado en un número de alumnos muy inferior -solamente ochenta y tres-. En cualquier caso, merece la pena señalar algunas de sus conclusiones. 
Al igual que el B789, las variaciones según el grupo de alumnos estaban muy presentes en las calificaciones del tutor del grupo nuclear y, en menor medida, en las calificaciones de la sesión de Introducción (y sorprendentemente en las calificaciones de la sesión de Preparación del Examen -una sesión para toda la escuela- y del director del curso). Las variaciones según las escuelas estaban presentes en las calificaciones de la sesión de Preparación del Examen y en las calificaciones de la disponibilidad del director del curso -y también en las calificaciones de la sesión de "Redacción de textos publicitarios" y de "WRS en acción".

La clasificación de los tutores en las sucesivas presentaciones del curso varía. Sin embargo, se puede decir que los tutores con calificaciones más elevadas y los tutores con calificaciones más bajas mantienen sus posiciones de un curso a otro.

La Tabla 10 que figura a continuación muestra las clasificaciones de la capacidad de los tutores durante tres presentaciones sucesivas del B794: primavera de 1996, otoño de 1996 y primavera de 1997.

Tabla 10. Clasificaciones de capacidad de los tutores del B794 presentaciones sucesivas

\begin{tabular}{c|c|c|c}
\hline Tułor & Primavera 96 & Oioño 1996 & Primavera 1997 \\
\hline A & - & 1 & - \\
\hline B & 1 & $2 / 3$ & $1 / 2$ \\
\hline C & - & $2 / 3$ & - \\
\hline D & - & - & 3 \\
\hline E & 2 & 4 & $5 / 6$ \\
\hline F & 3 & $7 / 8$ & \\
\hline G & 4 & 6 & $1 / 2 ; 7$ \\
\hline H & 5 & 5 & $4 ; 8$ \\
\hline I & 6 & $7 / 8$ & $5 / 6$ \\
\hline
\end{tabular}

\section{4. ¿INFLUYE LA CAPACIDAD DEL TUTOR EN LA VALORACIÓN GLOBAL DE LA ESCUELA?}

Una diferencia en la calificación de la capacidad del tutor de un punto en la escala está asociada a una diferencia en la valoración global de la escuela de medio punto en la escala.

Utilizando las cifras de la Tabla 7 deducimos la siguiente ecuación, que relaciona la calificación de la capacidad de un tutor con la calificación relativa a la valoración global de la escuela residencial. 
valoración global de la escuela $=2.7+0.4$ calificación de la capacidad del tutor .

Por ejemplo, si un tutor recibe la calificación máxima de 5, suponemos que la escuela estará calificada con 2.7 más 0.4 veces 5 , es decir, 4.7. Si un tutor recibe la calificación media de 3 , entonces suponemos que la escuela recibirá la calificación de 2.7 más 0.4 veces 3 , es decir, 3.9. De esta forma, la valoraciones globales reflejan en cierta medida las calificaciones del tutor, pero el abanico de las valoraciones globales es inferior que el de las calificaciones de los tutores. En realidad el abanico es solamente de cuatro décimas -lo sabemos por el número 0.4 de la ecuación anterior. El resultado aquí es muy significativo.

[Merece la pena hacer hincapié en que el análisis no implica que el factor sea exactamente 0.4 . Para reconocer nuestra incertidumbre, podemos pensar que el factor se encuentra en algún punto entre 0.2 y 0.6$]$.

Este análisis tiene dos problemas. En primer lugar, el resultado puede ser la influencia de un grupo de alumnos, más que la influencia de un tutor (aunque la regularidad de las calificaciones del tutor a lo largo de los años lo hacen menos plausible). El segundo problema es que la dirección de la influencia puede ser la contraria: puede resultar que la valoración global de los alumnos de la escuela puede trasladarse a sus calificaciones sobre su tutor, en lugar de lo contrario. Para evitar estos dos problemas, calculamos la influencia de la capacidad del tutor sobre la valoración global utilizando las calificaciones de la capacidad de los tutores de los años anteriores. Utilizando las calificaciones de la primavera de 1996 para predecir la valoración global de la primavera de 1997 obtenemos una ecuación que una vez más contiene el factor 0.4. Utilizando las valoraciones de tutores del otoño de 1995 y de la primavera de 1995 de forma similar obtenemos unos factores de 0.1 y 0.6 (cuya media es de 0.35 ). Debido al pequeño tamaño de la muestra, estos cálculos no son significativos. No obstante, tomados en conjunto, parecen sugerir un orden similar de magnitud al obtenido utilizando las calificaciones actuales del tutor para predecir la valoración global de la escuela.

El Apéndice 2 presenta algunas extravagantes especulaciones de café relativas a la repercusión de la capacidad del tutor sobre la realización de los exámenes. Llegan a la conclusión (a su manera loca y especulativa) de que el alcance de la capacidad del tutor en la escuela residencial tiene una influencia sobre el examen de más o menos un $1 \%$ en las puntuaciones.

\section{CONCLUSIÓN}

La investigación tenía por finalidad responder a tres preguntas. Por lo que se refiere a la primera de ellas, hemos descubierto que las escuelas residenciales del B789 y del B794 son de elevada calidad. Pasando al tema de la uniformidad del 
producto, hemos descubierto que las calificaciones permanecen notablemente estables entre los dos cursos y durante los cinco años transcurridos desde que se inició el B789. Parece existir una calidad bastante uniforme de provisión entre las distintas escuelas y entre el Reino Unido y Zimbabwe (aunque existe alguna evidencia, en función de las reacciones de los alumnos, de diferencias culturales entre los dos países). La tercera pregunta se refiere a los datos del tutor. Como cabía esperar, las diferencias entre tutores/grupos de alumnos se reflejan con más intensidad en las calificaciones de la capacidad de los tutores. Existe un moderado grado de regularidad en las calificaciones de un tutor de un año al siguiente. La capacidad del tutor parece tener alguna influencia sobre la valoración global de la escuela. No obstante, estas conclusiones deben tratarse con precaución, a la vista de las consideraciones metodológicas mencionadas en el Apéndice 3.

La finalidad más amplia de la investigación era ilustrar un planteamiento más sofisticado del análisis de los datos relativos a las escuelas residenciales. El paso conceptual fundamental adoptado fue pensar en términos de causa y efecto -en términos de cómo las entradas determinan los resultados. El segundo paso consistía en utilizar las pruebas estadísticas adecuadas para determinar si existía una relación significativa entre entradas y resultados específicos -y determinar el alcance de cualquier efecto significativo detectado. En sentido más amplio, se ha hecho hincapié en pensar cuidadosamente, a través de toda una serie de aspectos metodológicos (ver Apéndice 3).

Finalmente, debemos preguntarnos “¿y qué?”. Burt (1997, p. 134) argumenta que "por razones tanto lógicas como políticas existe la necesidad de demostrar que las evidencias que proporcionan las investigaciones institucionales tienen una repercusión sobre la organización”. El trabajo aquí reseñado fue redactado como Nota Estadística. Se envió un resumen del mismo a los integrantes del equipo del curso y a otra serie de personas de la universidad y se les invitó a que solicitasen una copia del informe completo. Cincuenta y una personas lo hicieron. Los comentarios fueron en general positivos. Merece la pena subrayar que los dos equipos del curso (B789 y B794) han concedido siempre mucha importancia a la evaluación de sus escuelas residenciales (y de sus cursos en conjunto). Siempre había detectado alguna cautela en relación con este ejercicio. Así, parte del interés de los equipos del curso se debió al hecho de que mis conclusiones apoyaban la validez de los datos:

"Por mi dinero, que lo que esto demuestra es que esta "hoja de la felicidad" que ha sido objeto de tantas burlas... puede producir, si se la toma en serio, información (sorprendentemente) significativa, incluso cuando se opera con pequeñas poblaciones. Creo que mucha gente dudaba que pudiesen resultar tan informativas (;me parece recordar un escepticismo bastante intenso por vuestra parte cuando iniciamos esta travesura hace cinco años!).

(presidencia del equipo del curso) 


\section{REFERENCIAS BILIOGRÁFICAS}

Brackenreg, J. and Allan. J. (1991). In defence of residential schools. Australian and South Pacific External Studies Association Papers, 11, 29-35.

BurT, G. (1997). Face to Face with Distance Education. Milton Keynes: Open and Distance Education Statistics.

Cameron, J., Davidson, G., Dekkers, J., Herrman, A. Livingston, K., Warner, L. and WilkinSON, J. (1991). On campus activities in the nation-wide provision of distance education. Evaluation and Investigations Program. Canberra: Department of Employment, Education and Training.

Cuskelly, E. and DeKKers, J. (1992). Student attendance and the costs of on-campus commitments for distance students. En: Evans, T. and Juler, P. Research in Distance Education 2. Geelong, Australia: Institute of Distance Education, Deakin University. 122-136.

EISEMAN, J. (1992). Redefining the role of residential schools in distance education. Distance Education Association of New Zealand Bulletin, 15, 45-52.

Eiseman, J. and Mahony, M.J. (1992). Alternatives to residential school: empowering students to succeed at home. En: Evans, T. and Juler, P. Research in Distance Education 2. Geelong, Australia: Institute of Distance Education, Deakin University. 178-189.

Gillie, A. and Woodley, A. (1995). Residential summer school attendance and students' assessed performances on Open University foundation courses. Internal paper. Milton Keynes: Open University.

Hermann, A., Cameron, J. and Davidson, G. (1991). On-campus requirements in remote area Australian distance education. Open Learning, 6,2,21-27.

KemBer, D. (1982). External sciences courses: the practical problem. Distance Education, 3, 2, 207-225.

McIntosh, N.E. (1975). The place of Summer Schools in the Open University. Teaching at a Distance, 3, 48-60.

Morgan, A. and Thorpe, M. (1993). Residential schools in open and distance education: quality time for quality learning? En Evans, T. and Nation, D. (eds.) Reforming open and distance education. London: Kogan Page, 72-87.

Parer, M. (1988). The cost of distance education. Cambridge International Extension College.

ReKkedal, T. LAmpiKoski, K. and VAn DER Mark, D. (1989). The AECS Survey 1988. Epistolodidaktika, 1, 8-17.

Rumble, G. (1997). The costs and economics of open and distance learning. London: Kogan page.

SiACIWENA, R.M.C. (1990). An evaluation of student support services at the University of Zambia. Open Learning, 5, 2, 19-23.

Timmins, K. (1989). Educational effectiveness of various adjuncts to printed study materials in distant education. Research in Distant Education, 1, 3, 12-14.

WARNER, L. and WiLKINSON, J. (1992). Evaluation of on-campus activities in disciplines necessitating compulsory attendance. Research in Distance Education, 4, 3, 2-5. 


\title{
PERFIL ACADÉMICO-PROFESIONAL DEL AUTOR
}

\author{
Mr Gordon Burt es profesor de tecnología Educativa en la Open University del Reino Unido. Está interesado por el análisis esta- \\ dístico y los modelos matemáticos relacionados con la educación abierta y a distancia, así como por los aspectos sociopolíticos de \\ la educación abierta y a distancia. Cuenta con recientes publicaciones no sólo en el campo de la educación a distancia y de los me- \\ dios educativos sino también en los campos de la política, la economía y la sociología. \\ Gordon Burt \\ Institute of Educational Technology \\ Open University \\ Walton Hall \\ Milton Keynes \\ MK7 6 AA \\ UK \\ Tel: 01908653846 (Oficina) 01908611296 (Particular) \\ Fax: 01908654173 \\ Correo Electrónico: g.j.burt@open.ac.uk
}

\section{APÉNDICE 1.}

¿Con qué grado de fiabilidad podemos calcular el efecto del grupo del alumno?

El análisis estadístico muestra que existe un efecto significativo asociado con el grupo del alumno. Sin embargo, este efecto no determina completamente la calificación de un alumno. La calificación del alumno contiene también un error aleatorio.

calificación de un alumno = el efecto del grupo + "error aleatorio"

El error aleatorio es responsable de al menos el 50\% de la varianza en las calificaciones de los alumnos de su tutor, y es responsable de al menos el 75\% de la varianza en las calificaciones de los alumnos de las sesiones y de su valoración global de la escuela.

Debido al error aleatorio nuestro cálculo del efecto del grupo del alumno contendrá un error aleatorio. De esta forma, podemos prever que la media de un tutor variará de una presentación a la siguiente.

Para ser más preciso, el error estándar de las medias de los tutores es a menudo de 0.2 o 0.3 . Digamos de 0.25. De esta forma, el intervalo de confianza es más o menos de 0.5. Por ello, podemos prever que la media del tutor recorra un punto entero de la escala. Nótese que esto concuerda con el comentario que hemos hecho de la Tabla 8, relativo a que los tutores con calificaciones en Capacidad de entre -0.5 y +0.5 no diferían significativamente de James Bond en 0 .

Uno de los motivos de que el error estándar sea tan grande es que el número de alumnos que contestan en cada grupo es pequeño -en algunos casos muy pequeño. La Tabla 9 que aparece más abajo muestra el número de alumnos que hay en cada grupo. Por ejemplo, había seis grupos que tenían once alumnos cada uno.

Tabla A1. Número de alumnos en cada grupo

\begin{tabular}{|l|r|r|r|r|r|r|r|}
\hline $\begin{array}{l}\text { № de alumnos en el grupo } \\
\text { № de grupos }\end{array}$ & 6 & 9 & 10 & 11 & 12 & 13 & 14 \\
\hline
\end{tabular}




\section{APENDICE 2.}

Extravagantes especulaciones de café relativas al efecto que tiene la capacidad del tutor de la escuela residencial sobre la realización de los exámenes

La finalidad de este artículo es suscitar la reflexión acerca de las magnitudes plausibles de los efectos que producen los diversos componentes del sistema OU. Habiendo obtenido un cálculo del éfeciu dé la capaciúaú úei iuiuı subıe la valoración global que los alumnos tienen de la escuela residencial, podemos proceder a reflexionar sobre la repercusión de la escuela residencial en la realización de los exámenes por parte de los alumnos.

Para comenzar, pensemos en el porcentaje de tiempo de estudio que los alumnos dedican a la escuela residencial. Supongamos que una unidad del curso y un día en la escuela residencial ocupan ambos diez horas. En este caso, doce unidades y dos días de escuela residencial implican que las escuelas residenciales ocupan el 14\% del tiempo de estudio de los alumnos. Si suponemos que la repercusión de un componente es proporcional al tiempo de estudio que se le dedica, esto implica que las escuelas residenciales deberín influir en un 14\% sobre las calificaciones del examen.

Este cálculo no tiene en cuenta el tiempo dedicado a la revisión -que por supuesto tiene lugar cuando se aproxima la fecha del examen y por ello puede tener mayor repercusión. Quizás deberíamos entonces reducir nuestro cálculo de la influencia de la escuela residencial sobre el examen a, por ejemplo, el 7\%. (Esta cifra está bastante próxima a la señalada por Alan Woodley y Allan Gillie en su estudio sobre las escuelas residenciales de hace unos años. Es el doble de la cifra de repercusión de las tutorías recogida por Burt (1997, p. 36-44)).

Nuestra cifra del 7\% es el efecto medio. Consideremos ahora cómo puede verse afectada por la variación de la capacidad del tutor. La capacidad del tutor recorre dos puntos de la escala. Aplicando nuestro factor de 0.4, lleva a un recorrido de 0.8 en las calificaciones globales de la escuela. Esto corresponde a una variación de más o menos 0.4 en relación con la calificación media de valoración global de la escuela, que es 4.4. Observando que la escala de calificación comienza en 1, esta variación corresponde a más o menos el $12 \%$ de la calificación media. El efecto de esta variación sobre el efecto medio del examen es de más o menos el $12 \%$ del $7 \%$, que grosso modo es más o menos el $1 \%$.

La conclusión de nuestra descabellada especulación de salón es que la escala de capacidad del tutor de la escuela residencial tiene una repercusión sobre las calificaciones del examen de más o menos el 1\%. ¡Bien! ¿Vds. qué opinan?

Desde luego, la página 4 del Manual de la Escuela Residencial del B789 supone que la escuela tiene tanto objetivos estrictamente relacionados con el curso como objetivos profesionales más amplios. Una ilustración de las posibles formas de investigación de la repercusión sobre objetivos educativos más amplios se ofrece en Burt (1997, pp. 51-62).

\section{APÉNDICE 3.}

\section{Aspectos metodológicos}

El presente estudio está basado en los 276 alumnos que asistieron a la escuela residencial del curso B789 correspondiente a la primavera de 1997 y que cumplimentaron el cuestionario de la escuela residencial. El curso tuvo lugar en cinco lugares distintos y requirió veinticinco tutores diferentes.

Los datos del cuestionario consisten en las respuestas de los alumnos sobre una escala de cinco puntos. Dicha escala no puede registrar más que diferencias muy crudas en los niveles de res- 
puesta. Tenemos también que ser cautos en relación con los aspectos de fiabilidad y validez. Además, los números que resultan del análisis serán estimaciones, sujetas a errores debidos al muestreo aleatorio y, posiblemente, a prejuicios.

\section{Otras variables}

Además de las variables seleccionadas para estudio, existen otras que tendrán una repercusión sobre medidas como la valoración global de la escuela. Es decir, que puede haber variables cuyos efectos no he verificado.

\section{Dirección de la causalidad}

En aquellos lugares en donde encontramos una relación hemos de tener en cuenta cómo debe explicarse: ¿las calificaciones del tutor provocan las de la escuela, o son las de la escuela (o los antecedentes de dichas variables) las que afectan a las del tutor? ¿O existe una causación recíproca, es decir, que las calificaciones del tutor influyen en las de la escuela y las de la escuela influyen en las del tutor?

\section{Combinación de efectos}

La relación observada ¿es atribuible a una combinación de efectos? Si es así, lo que observamos es el efecto neto o efecto medio. Los efectos del componente pueden ser de diferentes magnitudes y pueden consistir en efectos positivos o negativos. En particular, el efecto puede ser mucho mayor en alumnos particulares o en grupos de alumnos.

\section{Conclusión provisional}

Los resultados no nos permiten pronunciar un veredicto final. Para llegar a una conclusión provisional hay que llenar las lagunas del razonamiento utilizando juicios subjetivos y suposiciones arbitrarias.

\section{Magnitud del efecto}

Finalmente, hay que considerar cómo juzgar la magnitud de los resultados. ¿Qué importancia podemos esperar razonablemente que tenga la influencia de los tutores? Ver Apéndice 2.

\section{Carácter decisivo}

¿Qué importancia tiene el efecto? Sobre el "carácter decisivo” de un efecto observado, ver Burt (1997, pp. 48-49).

\section{Análisis estadístico}

En este apunte he intentado presentar los resultados de la manera más sencilla posible y evitando detalles técnicos. No obstante, es importante señalar que la validez del argumento depende, al menos en parte, de la validez del análisis estadístico que he llevado a cabo. Normalmente, la técnica utilizada ha sido el análisis de varianza. Además de esto, la nota a pie de página de la Tabla 9 cita algunos coeficientes de correlación. Finalmente, en la investigación de la relación entre la capacidad del tutor y la valoración global de la escuela se utilizó el análisis de regresión. 